\title{
Thermal rippling behavior of graphane
}

\author{
S. Costamagna, ${ }^{1,2}$ M. Neek-Amal, ${ }^{1,3,}{ }^{*}$ J. H. Los, ${ }^{4}$ and F. M. Peeters ${ }^{1}$ \\ ${ }^{1}$ Departement Fysica, Universiteit Antwerpen, Groenenborgerlaan 171, BE-2020 Antwerpen, Belgium \\ ${ }^{2}$ Facultad de Ciencias Exactas Ingeniería y Agrimensura, Universidad Nacional de Rosario and Instituto de Física Rosario, \\ Boulevard 27 de Febrero 210 bis, 2000 Rosario, Argentina \\ ${ }^{3}$ Department of Physics, Shahid Rajaee Teacher Training University, Lavizan, Tehran 16785-136, Iran \\ ${ }^{4}$ Institute of Physical Chemistry and Center for Computational Sciences, Johannes Gutenberg University Mainz, \\ Staudinger Weg 9, D-55128 Mainz, Germany \\ (Received 12 April 2012; published 23 July 2012)
}

\begin{abstract}
Thermal fluctuations of single layer hydrogenated graphene (graphane) are investigated using large scale atomistic simulations. By analyzing the mean square value of the height fluctuations $\left\langle h^{2}\right\rangle$ and the height-height correlation function $H(q)$ for different system sizes and temperatures, we show that hydrogenated graphene is an unrippled system in contrast to graphene. The height fluctuations are bounded, which is confirmed by a $H(q)$ tending to a constant in the long wavelength limit instead of showing the characteristic scaling law $q^{4-\eta}(\eta \simeq 0.85)$ predicted by membrane theory. This unexpected behavior persists up to temperatures of at least $900 \mathrm{~K}$ and is a consequence of the fact that in graphane the thermal energy can be accommodated by in-plane bending modes, i.e., modes involving C-C-C bond angles in the buckled carbon layer, instead of leading to significant out-of-plane fluctuations that occur in graphene.
\end{abstract}

DOI: 10.1103/PhysRevB.86.041408

PACS number(s): 72.80.Vp

Hydrogenated graphene (GE), called graphane (GA), is a quasi-two-dimensional (2D) structure of carbon (C) atoms ordered in a buckled honeycomb lattice covalently bonded to hydrogen $(\mathrm{H})$ atoms in an alternating, chairlike arrangement. ${ }^{1}$ Experimentally, it has been shown that GA can be obtained reversibly starting from a pure GE layer, ${ }^{2}$ and since then it has become a material of high interest due to its potential applications in nanoelectronics. ${ }^{3}$ As compared to GE, the chemisorption of the $\mathrm{H}$ atoms is accompanied by an important reconstruction of the chemical bonds and angles in the flat honeycomb lattice. ${ }^{4}$ Each carbon atom acquires an $\mathrm{H}$ neighbor, involving a transition from $s p^{2}$ to $s p^{3}$ hybridization, which turns the conjugated, graphitic C-C bonds into single C-C bonds, changing locally the planar shape of graphene into an angstrom scale out-of-plane buckled shaped membrane, ${ }^{5}$ as displayed schematically in Fig. 1.

One expects that at nonzero temperature thermally excited ripples will occur and distort the lattice. For GE these ripples can be described theoretically by the elasticity theory of continuum membranes. ${ }^{6}$ Among others, this membrane theory predicts a suppression of the long wavelength out-of-plane fluctuations by the anharmonic coupling between the out-ofplane bending and in-plane stretching modes, leading to a characteristic power law behavior for the height fluctuations as a function of the system size. Although the height fluctuations still diverge, the normal-to-normal correlation is preserved over a large length scale, stabilizing the membrane. By using atomistic simulations these results were shown to be applicable to GE, implying that this prototype $2 \mathrm{D}$ solid behaves as a membrane, ${ }^{7,8}$ and also to bilayer GE. ${ }^{9}$ Experiments have confirmed that suspended GE is not perfectly flat but instead presents ripples at finite temperatures. ${ }^{10}$

In this Rapid Communication we study thermally excited ripples in GA using state-of-the-art molecular dynamics (MD) simulations and show that the angstrom scale thermal ripples present in GE do not appear in GA for temperatures up to at least $900 \mathrm{~K}$. The $A$ - and $B$-sublattice buckling is preserved and inhibits the formation of long wavelength ripples. As a consequence, the calculated height-height correlation function $H(q)$ does not follow the characteristic $q^{4-\eta}$ power law scaling in the long wavelength limit predicted by membrane theory, and the height fluctuations appear to be bounded.

According to membrane theory and within the harmonic approximation, applicable in the short wavelength regime where $q$ is larger than some crossover value $q^{*}$, the outof-plane and in-plane modes are decoupled and the elastic bending free energy density is described by $F_{\text {harm }}=\kappa\left(\nabla^{2} h\right)^{2}$, where $h$ is the local height and $\kappa$ is the bending rigidity of the membrane which governs the properties of the temperature induced ripples. Substitution of the Fourier transform of $h$ and integrating over 2D space leads to the following height-height correlation function,

$$
H_{\text {harm }}(q)=\left\langle|h(q)|^{2}\right\rangle=\frac{N k_{B} T}{\kappa S_{0} q^{4}},
$$

where $N$ is the number of atoms, $S_{0}$ the surface area per atom, and $k_{B}$ is the Boltzmann constant. Accordingly, the height fluctuations in the harmonic regime behave as $\left\langle h^{2}\right\rangle_{\text {harm }}=C L^{2}$, with $C$ a temperature-dependent constant and $L$ the linear size of the system. In the large wavelength limit, i.e., for $q<$ $q^{*}$, the height fluctuations are suppressed by the mentioned anharmonic coupling between bending and stretching modes giving rise to a renormalized $q$-dependent bending rigidity $\kappa_{R} \propto q^{-\eta}$ and a power law scaling behavior

$$
H(q)=\frac{N k_{B} T}{\kappa S_{0} q^{4-\eta}},
$$

and accordingly $\left\langle h^{2}\right\rangle=C^{\prime} L^{2-\eta}$ with $C^{\prime}$ a constant $(\neq C)$. The universal scaling exponent $\eta$ has been estimated to be $0.821 .^{11}$ For GE, using Monte Carlo (MC) simulations with the empirical long-range carbon bond-order potential II (LCBOPII), ${ }^{12}$ good agreement with these results, derived 


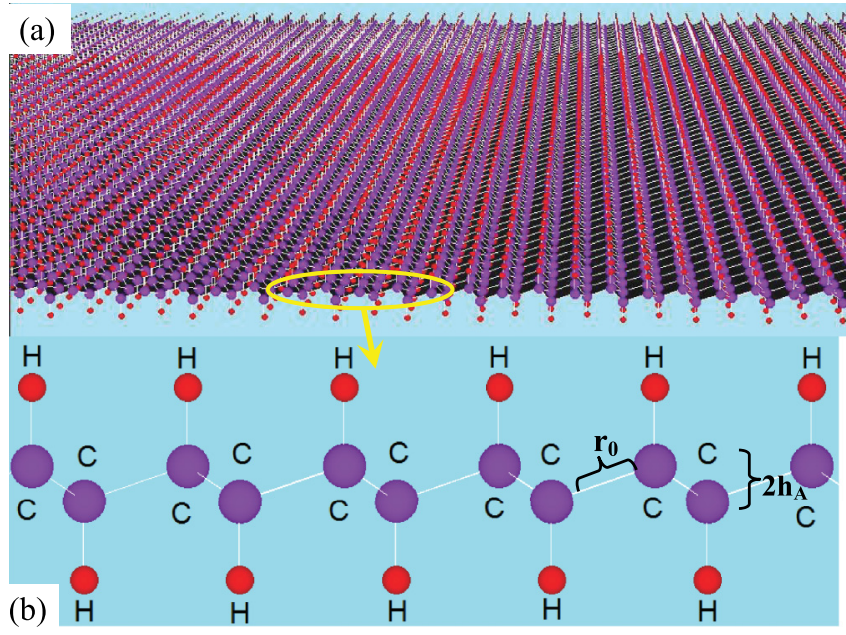

FIG. 1. (Color online) (a) Schematic view of a large sample of graphane. (b) Buckling between $\mathrm{C}$ atoms in the $A$ (higher) and $B$ (lower) sublattices at $T=300 \mathrm{~K}$.

from continuum theory, was found with $\eta \approx 0.85$. $^{7}$ Here, we investigate to which extent membrane theory can be applied to the description of thermally excited ripples in GA and we compare our results with those for GE.

To calculate the height fluctuations for GA we first need to define an appropriate value for the height $h_{i}$ of each lattice site $i$. Since we are mainly interested in the long wavelength fluctuations, which normally govern the size of the height fluctuations, we defined it on the basis of the carbon positions as

$$
h_{i}=\frac{1}{2}\left(z_{i}+\frac{1}{3} \sum_{j}^{\prime} z_{j}\right),
$$

where $i$ is a carbon atom, $\sum_{j}^{\prime}$ runs over the three carbon neighbors of $i$, and $z_{i}$ is the $z$ coordinate perpendicular to the plane. This definition allows for a straightforward comparison with GE, for which the heights were defined in the same way following previous work. ${ }^{9}$ To measure $\left\langle h^{2}\right\rangle$ and $H(q)$ we have performed MD simulations using the LAMMPS package. ${ }^{13}$ Both the GA and the GE systems have been sampled using the constant NPT ensemble (with $P=0$ ). For the interatomic interactions we used the modified second generation of Brenner's bond-order potential, i.e., adaptive intermolecular reactive bond-order (AIREBO) ${ }^{14}$ which has been shown to predict correctly the configurations for many different hydrocarbon structures. We simulated square shaped systems with the number of $\mathrm{C}$ atoms equal to $N=4860$, 8640, 19 440, and 37888 , in a temperature range from 100 to $900 \mathrm{~K}$ (note that for GA the total number of atoms is twice as large). Periodic boundary conditions were applied in the $x$ and $y$ directions. The presented results have been computed averaging over 300-400 uncorrelated configurations.

In Fig. 2 we show the out-of-plane positions for GE (a) and GA (b) for arbitrary snapshots taken during the simulation at room temperature. In GA, the $A$ and $B$ sublattices fluctuate around their mean heights $\left\langle h_{A, B}\right\rangle \cong \pm 0.256 \AA$. The scaling of $\left\langle h^{2}\right\rangle$ with the system size is displayed in Fig. 2(c). The obtained values for GE are in close agreement with previous reported MD results obtained with the reactive empirical
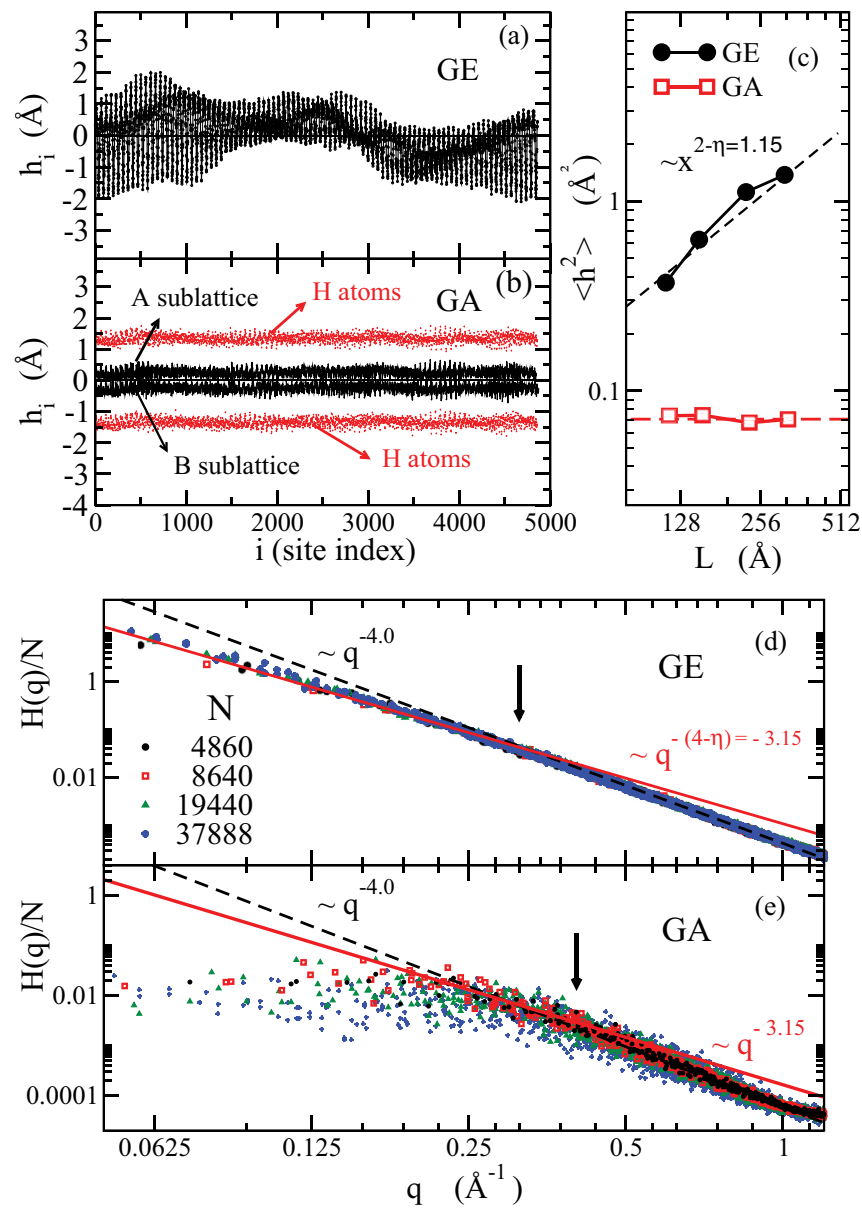

FIG. 2. (Color online) Heights of the $\mathrm{C}$ atoms in the GE (a) and GA (b) against the site index for arbitrary snapshots taken during the MD simulation. $N=4860 . T=300 \mathrm{~K}$. (c) $\left\langle h^{2}\right\rangle$ against $L=\sqrt{L_{x} L_{y}}$ in GE (circles) and GA (squares). $H(q)$ for different system sizes as indicated for (d) GE and (e) GA. The dashed line shows the harmonic $q^{-4}$ behavior and the solid line the correction due to anharmonic coupling for small $q$. Vertical arrows roughly indicate $q^{*}$ below which the harmonic behavior is broken.

bond-order (REBO) potential ${ }^{15}$ and slightly lower than those obtained from MC simulations using the LCBOPII. ${ }^{7}$ For GE, $\left\langle h^{2}\right\rangle$ increases as $L^{2-\eta}$ as expected from membrane theory. For GA, instead, $\left\langle h^{2}\right\rangle$ is almost independent of the system size. The differences in the rippling behavior of GA and GE are also evident from the results for $H(q)$ [Figs. 2(d) and 2(e)]. As it should be, the $H(q)$ functions for different system sizes overlap. However, for GA, although the harmonic $q^{-4}$ behavior for short wavelengths is well recovered, $H(q)$ tends to a constant in the long wavelength limit. Hence, it does not follow the $q^{4-\eta}$ power law as expected from membrane theory and found for GE.

The intermediate regimes where GE is only partially covered by $\mathrm{H}$ atoms are analyzed in Fig. 3(a). Notice that $H(q)$ displays harmonic behavior even for a $\mathrm{H}$ coverage as large as $90 \%$. The deviations at low wave vectors are small and hence can be ascribed to anharmonic coupling. The variation of $\left\langle h^{2}\right\rangle$, shown in the inset, indicates that first the sheet is softened when partially hydrogenated and becomes stiff at full coverage. 

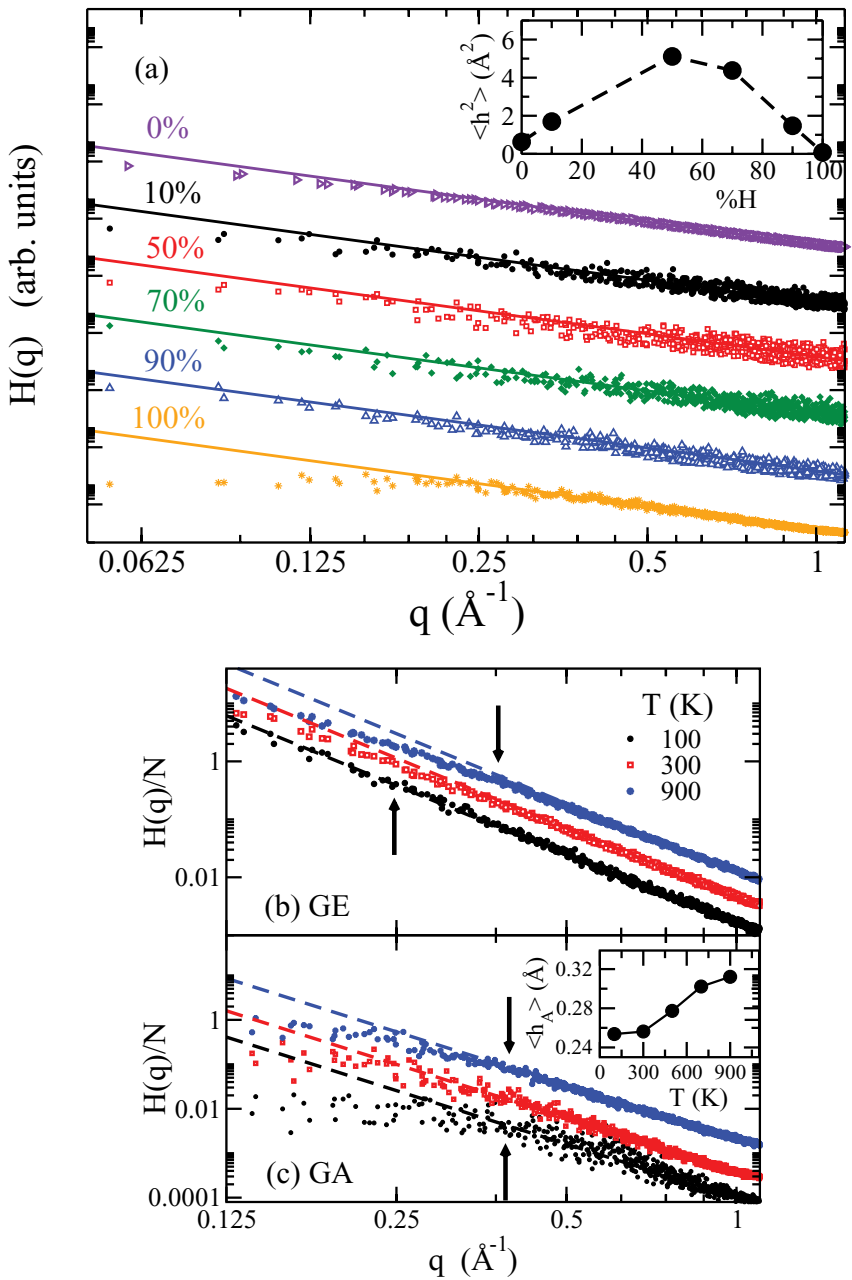

FIG. 3. (Color online) (a) $H(q)$ at $300 \mathrm{~K}$ for different $\%$ of $\mathrm{H}$ atoms ( $0 \%$ correspond to GE and $100 \%$ to GA). The different curves have been shifted for a better comparison. In the inset we show the variation of $\left\langle h^{2}\right\rangle . H(q)$ for different temperatures as indicated for (b) GE and (c) GA. The inset of (c) shows the average value of the heights in the $A$ sublattice of $C$ atoms against temperature. $N=8640$.

The behavior of $H(q)$ for different temperatures is shown in Figs. 3(b) and 3(c). As expected, with increasing temperature $H(q)$ is shifted to larger values for both GE and GA. In the inset of Fig. 3(c) we show the average heights of the $\mathrm{C}$ atoms in the $A$ sublattice against temperature. We also found that $\left\langle h_{A}\right\rangle \cong-\left\langle h_{B}\right\rangle$ over the whole temperature range, implying that the $A$ - and $B$-sublattice buckling is preserved.

More signals of the differences between the corrugations in GA and GE comes from the temperature dependence of $\left\langle h^{2}\right\rangle$ shown in Fig. 4(a). Note the difference in the vertical scale displayed on the left- and right-hand sides for GE and GA, respectively. While for GE the value of $\left\langle h^{2}\right\rangle$ changes about $0.61 \AA$ between 100 and $900 \mathrm{~K}$, the variation is only $0.07 \AA$ for GA, indicating that GA remains approximately unrippled even at $900 \mathrm{~K}$. The variation of $q^{*}$ against $T$ [Fig. 4(b)] also shows the same almost constant behavior for GA, whereas for GE, $q^{*}(T)$ behaves as expected for a 2D membrane. ${ }^{9}$ From the calculated $H(q \rightarrow \infty)$ at different $T$ and Eq. (1), one can also determine the $T$ dependence of the bending rigidity $\kappa$. Using the REBO potential it was found that $\kappa$ decreases with

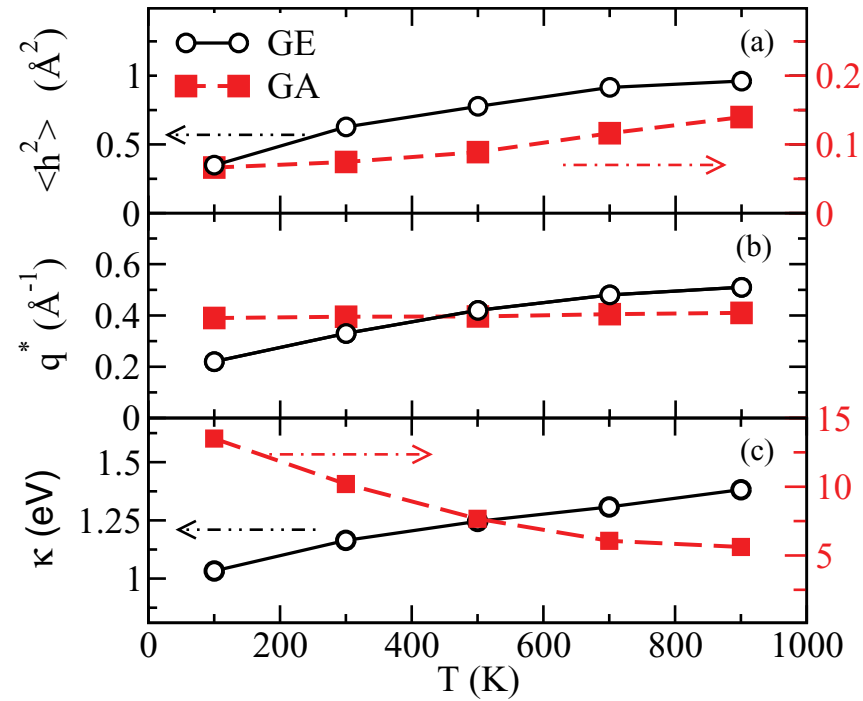

FIG. 4. (Color online) Variation of (a) $\left\langle h^{2}\right\rangle$, (b) $q^{*}$, and (c) $\kappa$ against temperature for GE (circles) and GA (squares). $N=8640$.

$T$ for $\mathrm{GE},{ }^{15}$ similarly as for liquid membranes. However, the opposite behavior was found using the LCBOPII potential. ${ }^{9}$ In Ref. 16 the rigidity was found to depend on the system size. Thus, the reported values for $\kappa$ in GE vary from 0.79 to $2.13 \mathrm{eV}$ depending on the calculation method. ${ }^{17}$ In Fig. 4(c) we show $\kappa$ for GE and GA calculated from the harmonic part of $H(q)$ between $q=0.5$ and $1 \AA^{-1}$, confirming that for GE, $\kappa$ increased with $T$. For GA, $\kappa$ is much larger and, most surprisingly, $\kappa$ strongly decays when temperature is increased, opposite to the behavior for GE.

The reason why GA does not obey membrane theory should be found in the geometry of the buckled carbon layer which

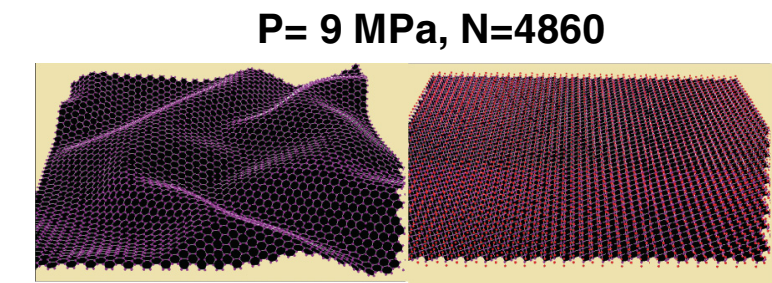

(b) GE

(c) GA

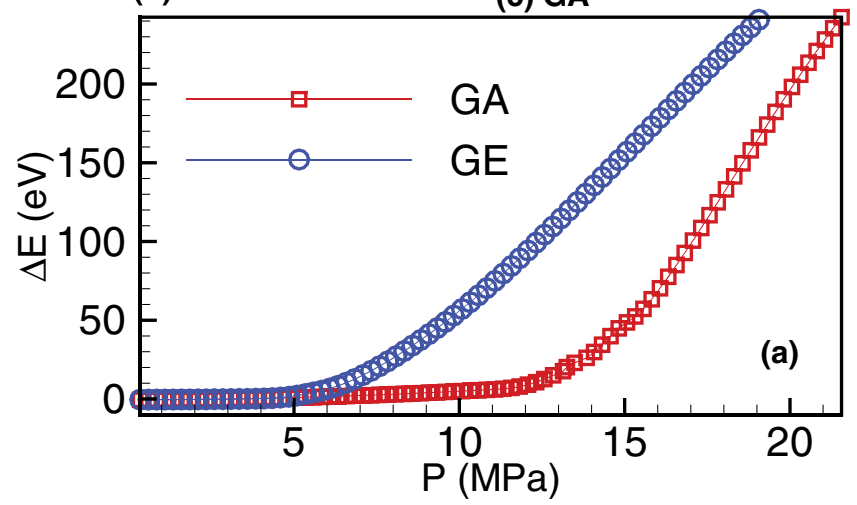

FIG. 5. (Color online) (a)Energy increase under biaxial pressure with respect to the relaxed system at zero pressure for GA and GE $(0 \mathrm{~K})$. Shape of (b) GE and (c) GA for $P=9 \mathrm{MPa}$. Note that while GE is corrugated, GA instead remains unrippled. 
allows for low energy in-plane bending modes, involving the $\mathrm{C}-\mathrm{C}-\mathrm{C}$ angles. These accordion types of modes have a relatively low energy. In principle this comes down to a strong anharmonic coupling of the out-of-plane bending mode with these in-plane bending accordion modes which strongly damp the out-of-plane excitations. To illustrate this further we have performed $N P T$ simulations for increasing pressure, the results of which are shown in Fig. 5. It shows that GA resists much higher pressures before bending than GE. ${ }^{18}$

Using MD simulations we have shown that the intrinsic thermal ripples present in GE do not appear in GA for temperatures up to at least $900 \mathrm{~K}$, which we ascribe to the angstrom scale buckling of the carbon layer in GA into a carbon bilayerlike configuration. The rippling behavior of GA is in disagreement with the continuum elasticity theory of membranes. The results from membrane theory are supposed to be universal, which means that they should not depend on the atomic scale details within the membrane. Instead, we find that GA can accommodate the thermal energy by in-plane bending modes, i.e., modes involving C-C-C bond angles in the buckled carbon layer instead of leading to significant out-of-plane fluctuations that occur in graphene. The present results for GA suggest that membranes of atomic scale thickness can exhibit a more complicated behavior than predicted by membrane theory.

We thank A. Fasolino, A. Dobry, and K. H. Michel for their useful comments. S.C. is supported by the Belgian Science Foundation (BELSPO). This work is supported by the ESF-EuroGRAPHENE project CONGRAN and the Flemish Science Foundation (FWO-Vl).
*Corresponding author: neekamal@ srttu.edu

${ }^{1}$ M. H. F. Sluiter and Y. Kawazoe, Phys. Rev. B 68, 085410 (2003); J. O. Sofo, A. S. Chaudhari, and G. D. Barber, ibid. 75, 153401 (2007).

${ }^{2}$ D. C. Elias, R. R. Nair, T. M. G. Mohiuddin, S. V. Morozov, P. Blake, M. P. Halsall, A. C. Ferrari, D. W. Boukhvalov, M. I. Katsnelson, A. K. Geim, and K. S. Novoselov, Science 323, 610 (2009).

${ }^{3}$ M. Z. S. Flores, P. A. S. Autreto, S. B. Legoas and D. S. Galvao, Nanotechnology 20, 465704 (2009); O. Leenaerts, H. Peelaers, A. D. Hernández-Nieves, B. Partoens, and F. M. Peeters, Phys. Rev. B 82, 195436 (2010); A. D. Hernández-Nieves, B. Partoens, and F. M. Peeters, ibid. 82, 165412 (2010); H. Sahin, C. Ataca, and S. Ciraci, ibid. 81, 205417 (2010); X. D. Wen, L. Hand, V. Labet, T. Yang, R. Hoffmann, N. W. Ashcroft, A. Oganov, and A. Lyakhov, Proc. Natl. Acad. Sci. USA 108, 6833 (2011).

${ }^{4}$ M. Neek-Amal and F. M. Peeters, Phys. Rev. B 83, 235437 (2011). ${ }^{5}$ D. W. Boukhvalov, M. I. Katsnelson, and A. I. Lichtenstein, Phys. Rev. B 77, 035427 (2008)

${ }^{6}$ D. Nelson, T. Piran, and S. Weinberg, Statistical Mechanics of Membranes and Surface (World Scientific, Singapore, 2004).

${ }^{7}$ J. H. Los, M. I. Katsnelson, O. V. Yazyev, K. V. Zakharchenko, and A. Fasolino, Phys. Rev. B 80, 121405 (2009).

${ }^{8}$ A. Fasolino, J. H. Los, and M. I. Katsnelson, Nat. Mater. 6, 858 (2007); S. Costamagna and A. Dobry, Phys. Rev. B 83, 233401 (2011); R. Roldán, A. Fasolino, K. V. Zakharchenko, and M. I.
Katsnelson, ibid. 83, 174104 (2011); M. Neek-Amal and F. M. Peeters, ibid. 82, 085432 (2010); Appl. Phys. Lett. 97, 153118 (2010).

${ }^{9}$ K. V. Zakharchenko, J. H. Los, M. I. Katsnelson, and A. Fasolino, Phys. Rev. B 81, 235439 (2010).

${ }^{10}$ J. C. Meyer, A. K. Geim, M. I. Katsnelson, K. S. Novoselov, T. J. Booth, and S. Roth, Nature (London) 446, 60 (2007); D. A. Kirilenko, A. T. Dideykin, and G. Van Tendeloo, Phys. Rev. B 84, 235417 (2011).

${ }^{11}$ P. Le Doussal and L. Radzihovsky, Phys. Rev. Lett. 69, 1209 (1992).

${ }^{12}$ J. H. Los, L. M. Ghiringhelli, E. J. Meijer, and A. Fasolino, Phys. Rev. B 72, 214102 (2005).

${ }^{13}$ [http://lammps.sandia.gov].

${ }^{14}$ S. J. Stuart, A. B. Tutein, and J. A. Harrison, J. Chem. Phys. 112, 6472 (2000).

${ }^{15}$ P. Liu and Y. W. Zhang, Appl. Phys. Lett. 94, 231912 (2009).

${ }^{16}$ Q. Wang, Phys. Lett. A 374, 1180 (2010).

${ }^{17}$ L. J. Karssemeijer and A. Fasolino, Surf. Sci. 605, 1611 (2011); K. H. Michel and B. Verberck, Phys. Rev. B 78, 085424 (2008); A. Lajevardipour, M. Neek-Amal, and F. M. Peeters, J. Phys.: Condens. Matter 24, 175303 (2012).

${ }^{18}$ See Supplemental Material at http://link.aps.org/supplemental/ 10.1103/PhysRevB.86.041408 for three movies which show the essential difference between thermal rippling behavior of graphene, partially covered graphene by hydrogens and graphane. 